\title{
The Growing N-Gram Algorithm: A Novel Approach to String Clustering
}

\author{
Corrado Grappiolo ${ }^{1}$, Eline Verwielen ${ }^{2}$, Nils Noorman $^{3}$ \\ ${ }^{1}$ ESI (TNO), Eindhoven, The Netherlands, corrado.grappiolo@tno.nl \\ ${ }^{2}$ Unaffiliated, Valkenswaard, The Netherlands, e_verwielen@hotmail.com \\ ${ }^{3}$ Philips Healthcare, Best, The Netherlands, nils.noorman@philips.com
}

Keywords: String clustering, n-grams, operational usage modelling, system verification testing.

\begin{abstract}
Connected high-tech systems allow the gathering of operational data at unprecedented volumes. A direct benefit of this is the possibility to extract usage models, that is, a generic representations of how such systems are used in their field of application. Usage models are extremely important, as they can help in understanding the discrepancies between how a system was designed to be used and how it is used in practice. We interpret usage modelling as an unsupervised learning task and present a novel algorithm, hereafter called Growing $\mathrm{N}$ Grams (GNG), which relies on n-grams — arguably the most popular modelling technique for natural language processing - to cluster and model, in a two-step rationale, a dataset of strings. We empirically compare its performance against some other common techniques for string processing and clustering. The gathered results suggest that the GNG algorithm is a viable approach to usage modelling.
\end{abstract}

\section{INTRODUCTION}

Connected high-tech systems allow the gathering of operational data at unprecedented volumes, usually in the form of log files. These, although historically mainly used for software debugging purposes, bring new opportunities to understanding how end-users are actually using a system, opposed to how they were designed to be used, for instance during system design. To be able to extract relevant log information and aggregate it into usage models can therefore be extremely beneficial. Usage models can help to identify particular types of behaviour, leading to insights which can serve as a basis for product improvements.

System verification can certainly benefit from usage models. During system verification, it is evaluated whether the system complies to regulations, requirements and specifications. System reliability verification is part of this process. The goal of reliability verification is to drive a system with realistic clinical scenarios in conditions which were not expected beforehand, taking care that the system is not loadand stress-tested. To evaluate the reliability of a system, its response has to be monitored at test time. If the response is not as expected, then the system has made a failure which may impact the operator or the patient. Reliability figures are usually expressed as mean time between failures, ranging from a few hundreds to thousands of hours. Ideally, the system is

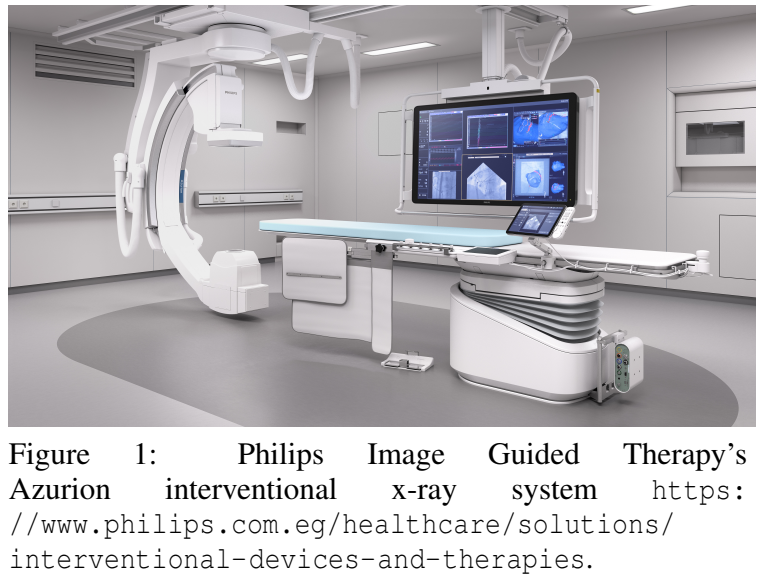

put under "average" use during reliability verification. However, it is difficult to determine what exactly the average use exactly is.

Our ultimate goal is the design of a computational framework for system (reliability) verification testing capable of distinguishing "average" usage (and other types of usage). Ideally, the framework would leverage usage models to identify, from a given dataset, logs depicting normal behaviour, and then to reexecute them. Additionally, such framework should be able to continuously maintain up-to-date representations of different behaviour types. We focus our attention on operational data of Philips Healthcare's 
Table 1: Example of an $\mathrm{x}$-ray sequence

\begin{tabular}{c||c|c} 
Step & Action & Symbol \\
\hline \hline 1 & table movement start & 1 \\
2 & x-ray arm movement start & 2 \\
3 & table movement stop & 3 \\
4 & x-ray arm movement stop & 4 \\
5 & table movement start & 1 \\
6 & table movement stop & 3 \\
7 & table movement start & 1 \\
8 & table movement stop & 3 \\
9 & user-interface & 5 \\
10 & user-interface & 5 \\
11 & user-interface & 5 \\
12 & x-ray acquisition start & 6 \\
13 & table movement start & 1 \\
14 & x-ray arm movement start & 2 \\
15 & table movement stop & 3 \\
16 & x-ray arm movement stop & 4 \\
17 & x-ray acquisition stop & 7
\end{tabular}

Image Guided Therapy interventional systems (IGT) - see Figure 1 - which make the task of modelling normal behaviour far from being trivial: the flexibility of this system, the medical staff's variety in educational background, their familiarity with manoeuvring the system and the different pathologies of the patients, result in an almost infinite amount of different usage types. Our interest, more specifically, is on the sequence of high-level actions performed by the users on the x-ray system during a medical procedure, rather than its low-level details, such as motor/sensor readings. Table 1 depicts an example of such $\mathrm{x}$-ray sequences.

Since we hold no a-priori knowledge on how many usage behaviours exist we interpret usage modelling as an unsupervised learning task. Assuming the successful partitioning of a given dataset into clusters, so that the similarity of behaviours represented by the string belonging to the same cluster is maximised, whilst the similarity of behaviours across clusters is minimised, the subsequent task would correspond to the extraction of a usage model for each cluster. Natural Language Processing (NLP) techniques are particularly useful. More specifically, we aim at extracting a n-gram model for each retrieved cluster. The main key-feature of n-grams is that they can lead to the creation of probabilistic graphs which can then be used for classification purposes (Jurafsky and Martin, 2014). In our approach, each node of a n-gram graph corresponds to a log action, whilst the edges connecting nodes correspond to the conditional probability of an action occurring given a finite sequence of previous actions.
Three main research questions arise: (1) how well do existing techniques used for string/text/document clustering perform on our data? (2) How can we compare the outcome of different clustering algorithms corresponding to a different number of clusters? (3) Is it possible to provide an alternative clustering approach?

To answer the first research question we considered $k$-means and complete-link hierarchical clustering — two of the most common clustering algorithms - in presence of raw text-data and string similarity measures or given a data transformation technique which converts the raw data into a vectorised form. The second research question is answered by leveraging on our modelling approach, which essentially aims to create probabilistic graphs for each cluster, to retrieve probability-based metrics - entropy in our case - to compare partitions obtained by using different algorithms and data transformation techniques. Concerning the third research question, we propose a novel algorithm, hereafter called Growing N-Grams (GNG). GNG creates new or updates existing n-gram models in a two-step rationale. First, given an input string to cluster, the algorithm relies on the existing models' symbols to identify the candidate models which share most commonalities with the string. If none are found, a new n-gram model based on that string is created. Otherwise, the algorithm calculates, for each candidate model, the chain probability of that string; the string is then assigned to the model which returned the highest probability, with the consequence that the winner model's n-grams and probabilities are updated to encompass the input string. To the authors' best knowledge, there has never been an attempt to perform string clustering directly by means of n-gram classification.

We conducted an empirical evaluation of string clustering based on a dataset describing subsequences of the Bolus chase procedure, a technique in peripheral arteriography, performed on Philips Healthcare's IGT interventional systems ${ }^{1}$. Once the set of n-gram models is built, the evaluation procedure consists of calculating the entropy score of the chain probabilities calculated by each n-gram model for a given validation set of strings. The rationale is the following: an optimal clustering partition would lead to n-gram models which do not "overlap", meaning that given an input string all but one model will return a very low chain probability, hence low entropy. On the other hand, the worst clustering partition would lead to n-gram models with some degree of overlap,

\footnotetext{
${ }^{1}$ The dataset, algorithms and additional content can be found using the following link: https://github.com/ bracciolo22/Growing-n-Grams.
} 
meaning that all models will return similar probability values, thus resulting in a high entropy score. By n-gram overlap we intend the existence of sub-graphs of nodes, edges and related probabilities, which are the same among different n-gram models, or at least very close to each other.

The gathered results suggest that: (1) GNG can outperform all other algorithms given appropriate hyperparameter setting; (2) there might be a relationship between the statistical properties of the dataset at hand and the hyperparameters leading to the optimal performance of GNG; (3) complete-link clustering with a simple string similarity measure such as the Levenshtein distance can lead to good results; (4) string vectorisation does not offer straightforward computational benefits. The investigation allows us to conclude that not only is GNG a novel, viable approach to string clustering, but its algorithmic structure is also suitable for our goal to create a framework for system (reliability) verification testing, given that it can continuously create new models and update existing ones, hence maintaining up-to-date representations of system usage, without the need to perform data pre-processing or pairwise distance matrix calculations.

The remainder of this document is organised as follows: Section 2 presents an overview of string clustering approaches existing in the literature. Section 3 formalises the problem at hand and the performance measure used for our empirical evaluation. Section 4 introduces the Growing N-Gram algorithm. Section 5 presents the experimental protocol adopted and the details regarding the algorithms considered. Section 6 describes and discusses the obtained results. Section 7 outlines some possible future work directions. Section 8 concludes the research.

\section{RELATED WORK}

One of the most popular approaches to text and document clustering focuses on converting the text data into a vectorised form, so that a similarity measure - mostly cosine similarity — can be computed and hence used by the most generic clustering algorithms, such as $k$-means (Bishop et al., 1995; Duda and Hart, 1973) and its numerous variants, or hierarchical clustering (Anderberg, 1973), in its agglomerative variation (Zhao et al., 2005; Jain and Dubes, 1988). Another term used to describe document vectorisation is bag-of-words (BOW). The idea is to leverage the statistical information of words populating a document to represent data in a vectorised form, most often retrieved by a combination of tech-

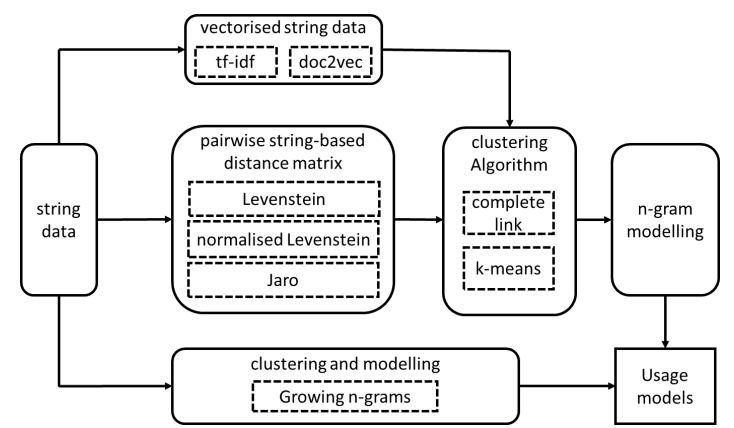

Figure 2: Clustering algorithms and data processing approaches considered in our study.

niques called Term Frequency and Inverse Document Frequency (tf-idf), (Raghavan and Birchard, 1979; Salton, 1989)). In essence, BOW abstracts documents as sets of words and ignores order dependencies. Hornik et al. (Buchta et al., 2012) utilise BOW and cosine similarity with spherical $k$-means (Dhillon and Modha, 2001); Steinbach et al. (Steinbach et al., 2000), instead, propose a new variant of $k$-means, called bisecting $k$-means, to cluster vectorised documents, and compare their results against agglomerative hierarchical clustering. Mahdavi and Abolhassani (Mahdavi and Abolhassani, 2009) propose another flavour of $k$-means - named harmony $k$-means - with the same data transformation techniques. Finally, Cutting et al. (Cutting et al., 2017) combine both $k$-means and hierarchical clustering in an algorithm called Scatter/Gather, although the underlying data processing part is very similar to the other previous work. Other related work are those of Balabantaray et al. (Balabantaray et al., 2015) on $k$-medoids and, most relevantly, of Zhao and Karypis (Zhao et al., 2005), which provide an extensive research on hierarchical clustering, both agglomerative and divisive, even by proposing a novel constrained version of it, on vectorised documents. Our work shares with all these the idea of using BOW with tf-idf for data vectorisation followed by $k$-means and hierarchical clustering. We differ from them on the nature of the data — strings rather than documents - and also on the fact that we are truly unaware of how many clusters exist, making the setting of $k$ much more difficult. For a broader overview of text clustering applications please refer to (Aggarwal and Zhai, 2012) and (Andrews and Fox, 2007) among other.

The recent raise in popularity and performance of neural-based computing - e.g. deep learning — has also contributed to NLP and therefore document clustering. Within this field, the vectorisation of documents and words is usually referred to as word embeddings (Dai et al., 2015). The key concept corre- 
sponds to train a neural network to predict a word given a contextual neighbourhood of text, and subsequently utilises only part of the trained network to convert an input text into a vector of real values. The context of a given word is mainly obtained by two similar techniques: skip-gram (Dai et al., 2015) and continuous-bag-of-words (Mikolov et al., 2013a), which both rely on a window of text around the given word, with the difference that skip-gram takes into account the order of words. Word embedding led to a vast plethora of work, see for instance Mikolov et al. (Mikolov et al., 2013b), Lau and Baldwin (Lau and Baldwin, 2016), Bengio et al. (Bengio et al., 2003), Kiros et al (Kiros et al., 2015), Kim (Kim, 2014), Zhang et al. (Zhang et al., 2015), Xu et al. (Xu et al., 2015), or Tai et al. (Tai et al., 2015), to name few. Intuitively, neural computing for word embedding is extremely popular and should not be ignored, that is why we considered it in our empirical evaluation. On the other hand, understanding the most consolidated approach or even network flavour is not a trivial task at all. We arbitrarily decided to rely on the work of Le and Mikolov (Le and Mikolov, 2014) — primarily for its popularity and practical ease of use of their algorithm - as an alternative approach to document vectorisation in combination with $k$-means and hierarchical clustering for benchmarking purposes.

As previously said, the data in our possession shares similarities also with sequences of strings with a predefined vocabulary. With this respect, there exist a vast plethora of string similarity measures which do not require any data transformation phase. Among these, the most widely known are arguably the Levenshtein or edit distance (Levenshtein, 1966) and variants (Pettersson et al., 2013; Ackroyd, 1980; Marzal and Vidal, 1993; Yujian and Bo, 2007), and the Jaro distance (Jaro, 1989). These metrics have been used, for instance, by Rajalingam and Ranjini (Rajalingam and Ranjini, 2011) in combination with agglomerative hierarchical clustering, albeit they pre-processed their text data by binary encoding; by $\mathrm{Lu}$ and $\mathrm{Fu}(\mathrm{Lu}$ and $\mathrm{Fu}, 1978$ ) in combination with $k$-nearest neighbour for sentence-to-sentence clustering; by da Cruz Nassif and Hruschka (da Cruz Nassif and Hruschka, 2013) in combination with partitional $k$-means, $k$ medoids and agglomerative clustering for partitioning filenames - hence strings of characters - in a forensic context. Another important relevant work is done by Wurzenberger et al. (Wurzenberger et al., 2017), who investigated several metrics for clustering $\log$ lines, though in a semi-supervised setting. Similarly to these work, we will embark the task of clustering our usage data by means of Levenshtein, normalised Levenshtein, and Jaro metrics.
N-grams (Chen and Goodman, 1999; Jurafsky and Martin, 2014; Kneser and Ney, 1995; Brown et al., 1992) - i.e. sequences of $n$ symbols - are extensively used in NLP, especially when combined with the Markov property (Markov, 1971), since they lead to the construction of probabilistic graphs. These allow for e.g. character and word prediction (Lesher and Rinkus, 2002; Lesher et al., 1998) and language modelling (Bungum and Gambäck, 2012; Siivola and Pellom, 2005). In accordance with these works, in fact, our ultimate goal is to model each cluster of operational data, depicting different usage behaviours, into a n-gram model. Attempts to leverage n-grams for text clustering have also been done. Ismail and Rahman (Ismail and Rahman, 2014) use the chain probability computed on tri-grams to calculate the similarity score of word pairs, and then decide whether to cluster them together based on a thresholding rationale. Kešelj et al. (Kešelj et al., 2003), instead, rely solely on the most frequent n-grams to calculate the similarity between document author profiles; similar strategy is adopted by Miao et al. (Miao et al., 2005), where they use most frequent n-grams as distances to be used by $k$-means for document clustering. Although our approach shares the use of chain probabilities and thresholding to determine whether a string should belong to a model, the n-grams are used in their entirety rather than just their most frequent terms, they are incrementally built through the assignment of new strings to related clusters, and finally no other clustering algorithm such as $k$-means is used.

\section{PROBLEM STATEMENT}

This section formally introduces the problem at hand, the strategy to solve it, and the performance measure used to evaluate the solutions found. In order not to overcrowd the manuscript, only the most important concepts are presented. Finally, in order to maintain the mathematical notions as simple and as clear as possible, sub- and super-script indices will be omitted when not necessary.

Let $\mathcal{D}$ be a dataset composed of $|\mathcal{D}|=d$ strings $w_{i}, i=1 \ldots d$. Each string $w$ is composed of a variable number of characters or symbols $x$ belonging to a vocabulary $\mathcal{V}$. $\mathcal{V}$ is retrieved from $\mathcal{D}$. $\mathcal{D}$ can contain more than one occurrence of a string $w$, and each string can contain more than one occurrence of the same symbol. The goal is to partition $\mathcal{D}$ into $k$ clusters $C_{i}, i=1, \ldots k$ and to build, for each cluster $C_{i}$, a n-gram $G_{i}$ solely based on the strings belonging to $C_{i}$. All n-gram models share the same value for $n$, that is 
the history of past events to be taken into account. No information is known, a priori, about $k$. We will refer to the set of n-gram models $M=\left\{G_{1}, G_{2}, \ldots G_{k}\right\}$ as an ensemble of models. Given a string $w$ composed of a sequence of $m$ symbols $w=x_{1}, x_{2}, \ldots x_{m}$ and an n-gram model $G$, the chain probability of $w$ given $G$ and $n$ is calculated as follows:

$$
\begin{gathered}
P(w \mid G)=P\left(x_{1}, x_{2}, \ldots x_{m} \mid G\right) \approx \\
\quad \approx \prod_{i=1}^{m} P\left(x_{i} \mid x_{i-1}, \ldots x_{i-n+1}, G\right)
\end{gathered}
$$

therefore, the assignment of $w$ to a n-gram model $G$ is obtained as follows:

$$
G^{\text {win }}=\underset{G}{\arg \max }(P(w \mid G), \forall G \in M)
$$

and, consequently, the corresponding cluster $C^{\text {win }}$ is also retrieved. As we will show in Section 5, different algorithms - or even the repeated execution of the same algorithm - might lead to a different number of clusters. In order to evaluate how well the partitioning task is performed, given an input string $w$, we calculate the entropy of the ensemble's chain probabilities:

$$
\mathcal{H}(w)=-\sum_{i=1}^{k} P\left(w \mid G_{i}\right) \log P\left(w \mid G_{i}\right)
$$

The rationale is the following: a good partition would lead to an ensemble which, for a given $w$, would return very low chain probability values for all-but-one model $G$ - i.e. the model obtained from Equation 2 - which instead would return a very high probability. Such a set of probabilities would lead to an extremely low entropy. On the other hand, a bad partitioning would lead to an ensemble in which all models return chain probability values which are close to each other, hence a high entropy score.

Given a set of ensembles $\mathcal{M}=\left\{M_{1} M_{2} \ldots M_{m}\right\}$ resulting from a clustering-modelling task of $m$ different algorithms, and a validation set $\mathcal{W}$ composed of $|\mathcal{W}|=l$ strings, the best ensemble is defined as follows:

$$
M^{*}=\underset{M}{\arg \min }\left(\frac{1}{l} \sum_{i=1}^{l} \mathcal{H}_{M}\left(w_{i}\right)\right), \forall M \in \mathcal{M}
$$

that is, it is the ensemble which returns the lowest average entropy score (and lowest standard deviation) calculated over the whole validation set.
The Growing N-Gram Algorithm

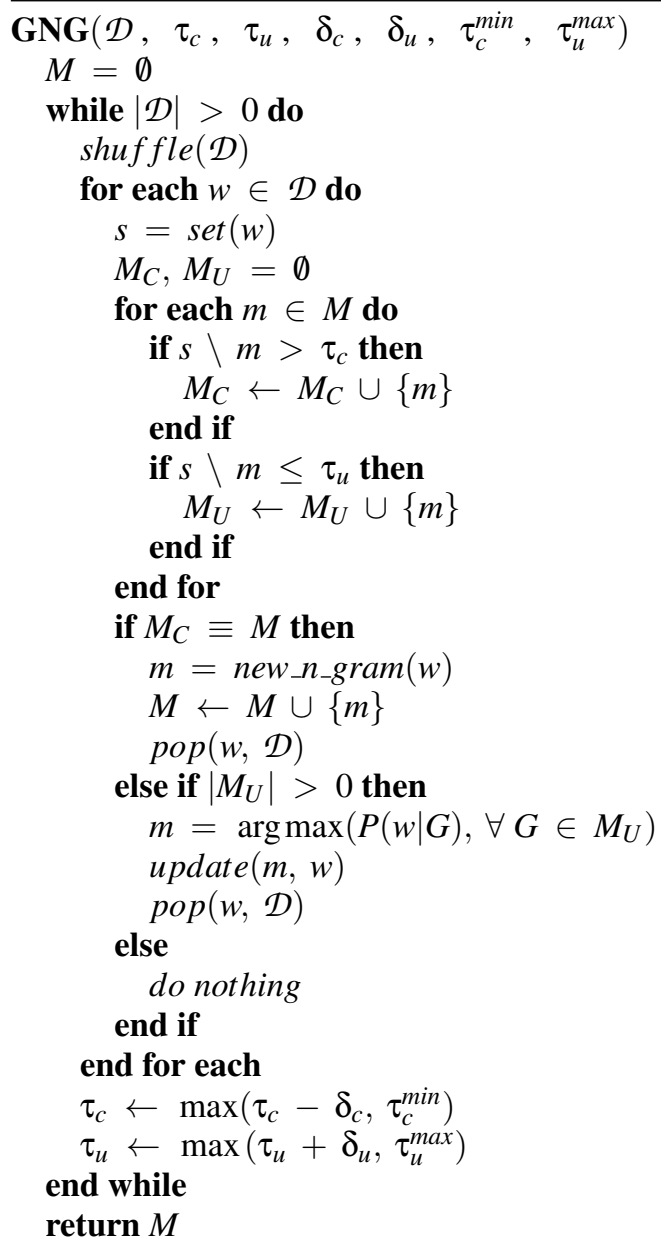

\section{THE GROWING N-GRAM ALGORITHM}

The GNG algorithm is presented as pseudo-code in the next column of this page. The algorithm takes as input: (1) a dataset $\mathcal{D}$ of strings to be partitioned into clusters, (2) two thresholds, namely $\tau_{c}$ and $\tau_{u}$, which regulate the creation of new models or the update of existing ones, (3) two parameters $\delta_{c}$ and $\delta_{u}$, which modify the values of their respective $\tau_{\mathrm{s}}$ so that the whole dataset is processed, (4) two parameters $\tau_{c}^{\min }$ and $\tau_{u}^{\max }$, which are used to bound the values of $\tau_{c}$ and $\tau_{u}$ respectively.

GNG assigns each string $w \in \mathcal{D}$ to one and only one model $m \in \mathcal{M}$, see lines 17 and 22. The pop function call in lines 19 and 23 simply means that $w$, once assigned to a model $m$, is removed from $\mathcal{D}$. For simplicity, the algorithm does not explicitly state how 
Table 2: Dataset Details

\begin{tabular}{c||c|c|c|c} 
Feature & Train & Test & Validation & Total \\
\hline \hline Size & 5301 & 663 & 662 & 6626 \\
Unique Strings & 1673 & 281 & 310 & 2019 \\
String Intersection with Training Set & & 113 & 131 & \\
\hline Alphabet Size & 88 & 57 & 61 & 91 \\
Alphabet Intersection with Training Set & & 56 & 59 & \\
\hline Avg. String Length & 7.18 & 7.93 & 7.82 & 7.32 \\
Std. Dev. String Length & 13.28 & 26.92 & 12.11 & 15.11 \\
Median string Length & 4 & 4 & 4 & 4 \\
Min. String Length & 2 & 2 & 2 & 2 \\
Max. String Length & 345 & 617 & 148 & 617
\end{tabular}

to maintain the cluster composition of each model. For each $w$, the algorithm first extracts the set $s$ of its unique symbols (line 6), then evaluates it with the symbols of each $m \in M$. The evaluation is done via set difference and this is compared against the two thresholds $\tau_{c}$ and $\tau_{u}$ (lines 9 and 12 respectively). Two subsets of $M, M_{C}$ and $M_{U}$, are used to keep track of which model satisfies which threshold condition lines 10 and 13 respectively. Please note that $M_{C}$ and $M_{U}$ do not partition $M$, meaning that it is possible that some model in $M$ might belong neither to $M_{C}$ nor to $M_{U}$ during a GNG iteration.

In essence, $M_{C}$ corresponds to the set of models in $M$ which are too far from $w$, whilst $M_{U}$ are the set of models in $M$ which are close enough to $w$. If all existing models are too far from $w$ (line 16), then a new n-gram model based on $w$ is created (line 17) and added to the ensemble (line 18). Alternatively, and in case there are some models which are close enough to $w$ (line 20), the model in $M_{U}$ which returns the highest chain probability calculated in accordance with Equation (2) is retrieved and updated with $w$ (lines 21 and 22). In case no creation or update is performed, the string remains unassigned (line 25) and the algorithm continues by processing the next string. The update of an n-gram model simply corresponds to updating the n-gram counts of that model given $w$. The consequence of this is that the calculation of the model's chain probabilities will also become up-to-date (Jurafsky and Martin, 2014; Kneser and Ney, 1995).

At the end of one dataset processing loop, some strings might still be unassigned. This is because the constraints based on $\tau_{c}$ and $\tau_{u}$ might be too strict. The algorithm then relaxes such constraints in lines 28 and 29. $\tau_{c}$ is decreased, whilst $\tau_{u}$ is increased. Both are bounded by their respective $\tau_{c}^{\min }$ and $\tau_{u}^{\max }$. $\mathcal{D}$ is guaranteed to be fully partitioned into clusters/models if, eventually, $\tau_{c}=0$ or $\tau_{c}=\tau_{u}$.

\section{EXPERIMENTAL PROTOCOL}

In order to evaluate the viability of our GNG algorithm we conducted an empirical investigation based on sub-sequences of the Bolus chase procedure, a technique in peripheral arteriography, performed on IGT systems. For optimal handling, we represented the log data as tokenised strings based on a vocabulary $\mathcal{V}$ of actions extracted from the dataset. An example of $\log$ tokenisation is depicted in Table 1 . We gathered and tokenised $6626 \mathrm{x}$-ray sequences leading to a vocabulary of 91 symbols. It is important to remark that each string $w$ can only terminate with one out of four possible symbols representing the end of an X-ray exposure type. The statistical details of our dataset are reported in Table 2. As it can be observed, the dataset contains a lot of repeated sequences - only $30 \%$ of instances are unique, which indeed gives us a hint that there must exist some usage patterns, though the string overlap between train and test/validation set is minor $(40.21 \%$ and $46.62 \%)$. On the other hand, all sets share most of the symbols.

Given the first research question introduced in Section 1, and the related work presented in Section 2, we decided to compare our GNG algorithm with several others, based on the following:

- data representation: we considered both the case in which each $w \in \mathcal{D}$ is vectorised or not;

- string vectorisation: we considered two techniques: (1) tf-idf and (2) Gensim's implementation of the doc2vec algorithm (Rehurek and Sojka, 2010). The similarity score of vectorised strings is calculated via cosine distance;

- non-vectorised data: in order to calculate the similarity of two strings we considered: (1) the Levenshtein distance, (2) the normalised Levenshtein distance, and (3) the Jaro distance;

- clustering algorithms: we considered $k$-means and complete-link hierarchical clustering; 


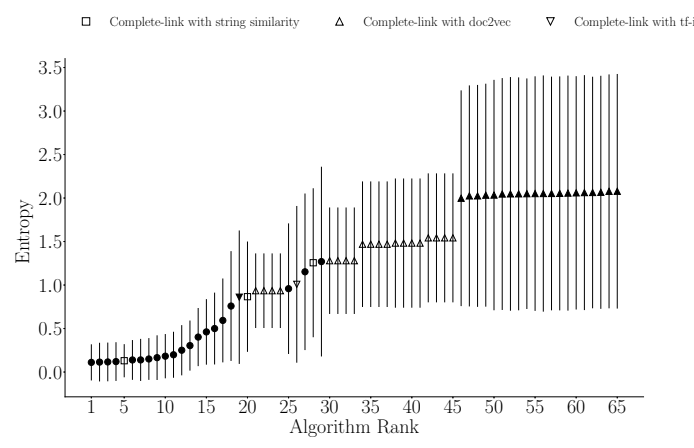

(a) Algorithm performance

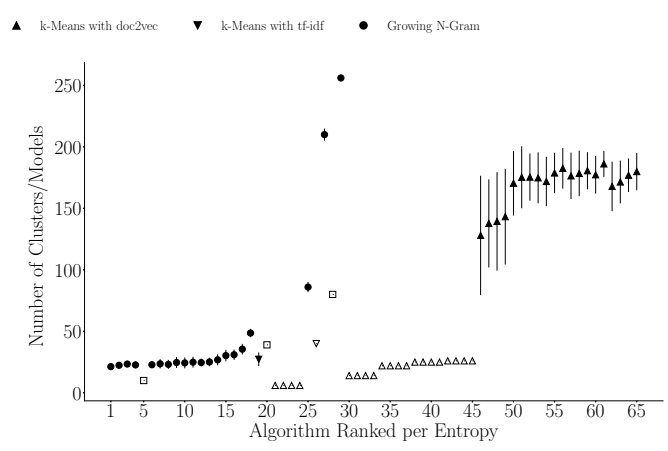

(b) Number of clusters/models per algorithm

Figure 3: Overview of the clustering algorithms considered in our investigation. Fig. (3a): average and standard deviation entropy scores per algorithm; Fig. (3b): average number and standard deviation of clusters/models per ranked algorithm.

- determining the number of clusters for $k$-means: we run $k$-means for $k=2, \ldots k^{\max }$ and rely on the silhouette coefficient in order to retrieve the best $k$-value.

- determining the number of clusters for completelink: we rely on the elbow rule calculated on the percentage of variance unexplained for $k=2, \ldots k^{\max }$. The elbow is retrieved by performing a two-segment curve fitting task; the value $k$ is the one which minimises the mean square error between the percentage of variance unexplained curve and the fitted segments.

- n-gram modelling: we rely on Kneser-Ney smoothing (Kneser and Ney, 1995). This is also implemented for GNG.

We decided to rely on generic forms of $k$ means and complete-link in order to have a broad overview of the performance that these algorithms could achieve. Similarly, Gensim's doc2vec was chosen due to its popularity in the neural computingbased NLP community. A summary of the approaches and algorithms considered can be observed in Figure2. Moreover:

- we partition $\mathcal{D}$ into train-test-validation sets $80 \%, 10 \%$ and $10 \%$ of $\mathcal{D}$ respectively. The sets are defined a priori and are the same for all algorithms and their repetitions. The train set is used by all algorithms to perform the clustering task. $k$ means relies on the test set to identify the value for $k$ via silhouette score. The validation set is used to identify the best algorithm/ensemble in accordance with Equation (4);

- all $k$-means and GNG instantiations are repeated 30 times. The same is done for complete-link in combination with doc2vec, whilst for the other complete-link settings, given their deterministic characteristics, only one repetition is performed;
- doc2vec is implemented with: train epoch 100 , vector size $v s \in\{2,4,6,8,10\}$, window size $w s \in\{2,5,10,20\}, \alpha=0.025$ for a total of 20 possible settings;

- for both $k$-means and complete-link, $k^{\max }=200$;

- for GNG, we investigated $\tau_{c} \in\{1, \ldots 20\}$, $\tau_{u}=-1, \delta_{c}=1, \delta_{u}=0.5, \tau_{c}^{\min }=\tau_{u}^{\max }=1 ;$

- $n=2$ for all $n$-gram models.

In total, therefore, we considered 20 settings for GNG, 21 settings for $k$-means (20 for doc2vec), 24 settings for complete-link (20 for doc $2 \mathrm{vec}$ ), resulting in 65 algorithmic settings.

\section{RESULTS AND DISCUSSION}

Figure $3 \mathrm{a}$ depicts the average and standard deviation entropy scores of the 65 algorithmic settings. The algorithms are ranked by average entropy score increasing. Once again, the lower the entropy score, the better the classification task, hence the clustering task. Furthermore, Figure 3b depicts the average and standard deviation number of clusters/models returned by each algorithm ordered in accordance with the rank of Figure 3a.

17 out of the first 18 best ranked algorithms are instances of GNG. The $\tau_{c}$ values range from $\tau_{c}=20$, which achieves the best average entropy score of 0.1109 (Std. Dev.=0.2077), to $\tau_{c}=4\left(18^{\text {th }}\right)$, with average score 0.7587 (Std. Dev. $=0.6311)$. Interestingly, $\tau_{c}=20$ resembles the average plus standard deviation string length for both train and validation set (20.46 respectively 19.93), whilst $\tau_{c}=4$ corresponds to the median string length for both train and validation set - see Table 2. A Welch's t-test with $\alpha=0.01$ under the null hypothesis that GNG with $\tau_{c}=20$ has the same average performance as 


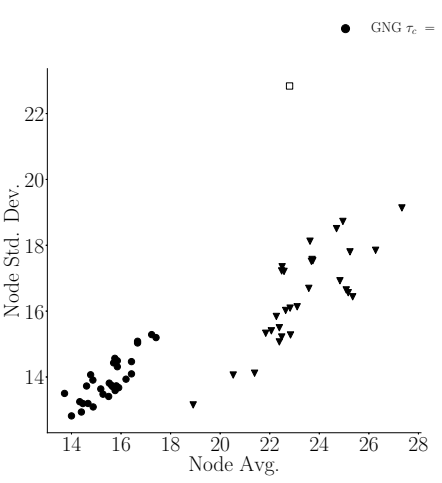

(a) Number of Nodes

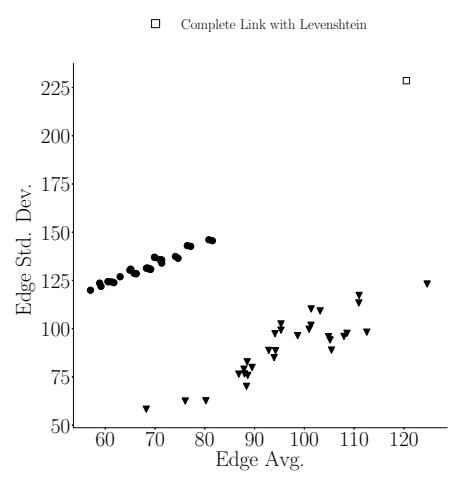

(b) Number of Edges

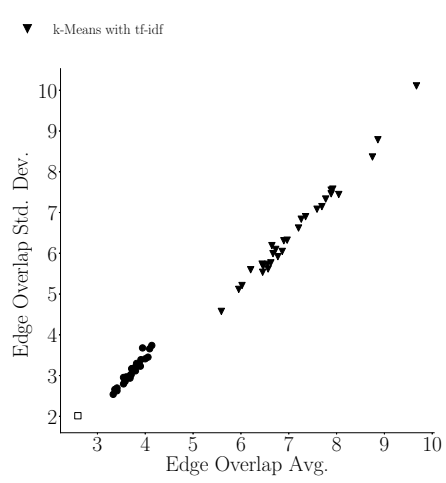

(c) Edge Overlap

Figure 4: Distribution details of n-gram model sizes and overlap for each of the three algorithm's best performance: Fig.( 4a) number of nodes, Fig. $4 \mathrm{~b}$ number of edges, Fig. $4 \mathrm{c}$ edge overlap across models over 30 algorithmic iterations.

the other algorithms lead to a p-value which make us reject such hypothesis from the fourth ranked algorithm - i.e. GNG with $\tau_{c}=18$ onward (the p-value for such comparison is $0.00001675, t=-4.3048$, $d f=39718)$. An example of a mid-to-large model obtained via GNG with $\tau_{c}=20$ can be seen in Figure 5.

Complete-link resulted in the second best performing algorithm. Strikingly, when complete-link is combined with the Levenshtein distance, its performance corresponds to the fifth best result, with an average entropy score of 0.13 (Std. Dev. = 0.1905). Another remarkable finding is that complete-link, when coupled with doc2vec, resulted in average (and Std. Dev.) entropy scores which were solely dependent on the vector size parameter. In other words, given e.g. $v s=2$, complete-link for all $w s \in\{2,5,10,20\}$ scored an average entropy of 0.9349 (Std. Dev. = 0.4289). This is observed for all $v s \in\{2,4,6,18,10\}$. The independence on $w s$ for complete-link given $v s$ is also observed in terms of average number of clusters retrieved, see Figure $3 \mathrm{~b}$. It is hard to find a plausible explanation of such behaviour. Although one might expect results along these lines for large $w s$ values given the string length statistics of our dataset, it is not clear why the same is also achieved for $w s=\{2,5\}$ as well. Further investigations aimed at shedding light on such results are encouraged, even beyond the case study considered here.

Another key-finding is the poor performance that $k$-means achieves when coupled with doc 2 vec. The 20 settings correspond to the 20 worst ranked performances and, unlike what was observed for completelink, it is not possible to find correlations between $v s$, $w s$ and the entropy scores. Moreover, the recorded standard deviations are the biggest across all algorithms considered. An explanation could be that doc2vec essentially adopts a bag-of-word vectorisation approach, hence ignores the order of symbols in strings, which is in fact a fundamental property of our data. This is also partly observed by the performance of both $k$-means and complete-link combined with tf-idf (ranked $19^{\text {th }}$ and $26^{\text {th }}$ respectively). Another possible explanation could be that doc2vec might require many more training epochs in order to provide reliable embeddings: the rank of complete-link with doc2vec is in fact ordered by $v s$ increasing, which also corresponds to a higher number of neurons in the embedding models, therefore more weights to be trained. Along these lines, one might argue that doc $2 \mathrm{vec}$ requires much bigger datasets.

To conclude, by looking at Figure $3 b$, we can observe that there is no correlation between the number of clusters and classification performance. This supports our claim that the average entropy score is a viable measure for the evaluation of clusters of strings when these can be generalised by probabilistic models.

We now centre our attention on analysing why GNG with $\tau_{c}=20$ has led to the best entropy scores, as opposed complete-link with Levenshtein distance and $k$-means setting with tf-idf, i.e. the two best alternative benchmark algorithms. The performance details can be found in Table 3. Figure $4 a$ depicts the average and standard deviation of nodes of the resulting ensembles across each of the 30 iterated runs; Figure $4 \mathrm{~b}$ depicts the same type of information, albeit about the number of edges composing the n-gram models, and finally Figure 4c depicts the average and standard deviation number of edges which are shared across models of the same ensemble. Again, n-gram models can be considered as probabilistic graphs, where the nodes corresponds to unigrams/unique symbols of the n-gram, and the edges correspond to the probability of observing a symbol 
Table 3: Algorithm Performance - Selected Details

\begin{tabular}{c||cc|cc} 
Rank & Algorithm & Parameters & Avg. Entropy (Std. Dev.) & Avg. Num. Clusters (Std. Dev.) \\
\hline \hline 1 & GNG & $\tau_{c}=20$ & $0.1109(0.2077)$ & $21.3(2.33)$ \\
5 & Complete-link & Levenshtein & $0.13(0.1905)$ & $10(0)$ \\
19 & $k$-means & tf-idf & $0.8603(0.767)$ & $27.3(5.53)$
\end{tabular}

given a previous one, since in our investigation we consider $n=2$.

By observing Figures $4 \mathrm{a}$ and $4 \mathrm{~b}$, we can see that GNG leads to ensembles composed of generally smaller models than complete-link and $k$-means, both in terms of nodes and edges composing them. The standard deviation values of the nodes is also smaller than the other two algorithms, and although this is not seen for edges, the range of GNG's standard deviation is much smaller than the other two algorithms. This also means that GNG is more robust against the randomisation of the dataset, which also sets the initial conditions of the algorithm. The high standard deviation in edge distribution suggests that GNG generates ensembles where some models have many edges and some others less. Such behaviour, although much stronger in magnitude, is also observed for completelink, as opposed to the $k$-means setting.

By looking at Figure 4c, moreover, we can see how little edge overlap is obtained by complete-link and GNG, as opposed to $k$-means, which also supports our assumption that low model overlap leads to low entropy scores. Strikingly, complete-link results in ensembles with a lower edge overlap than GNG, yet its entropy score is significantly worse. A plausible explanation could be that complete-link, being based on Levenshtein distance, calculates string similarities solely based symbols (hence graph nodes), as opposed to GNG, which instead takes into account both nodes (for new model creation) and edges (for model update). This might make complete-link more focused on the symbols in strings rather than sequences of symbols, possibly leading to a higher degree of overfitting: during validation, complete-link would face more sequences with out-of-vocabulary symbols than GNG, hence leading to low probabilities across different models, hence similar chain probability values, hence higher entropy scores than GNG. GNG with slightly higher edge overlap, on the other hand, is able to generate models which leave room for slightly different sequences, hence more generic ones, which would lead to lower entropy scores during validation.

\section{FUTURE WORK}

The results presented in the previous Section suggest that our GNG algorithm is a viable approach to string clustering and modelling. However, in order to better understand how generic and robust our algorithm is, a vast amount of research is still required. We here outline what we consider being the most salient topics of investigation.

First and foremost, GNG should be tested against different dataset types. An immediate investigation would correspond clustering full medical procedures rather then their sub-sequences. GNG should also be tested in the presence of a smaller/larger vocabulary, and possibly even towards pure text data, rather than sequences of symbols.

A thorough investigation on GNG's hyperparameters is due, as well as considering other settings for the benchmark algorithms considered. We believe that understanding the role of $\tau_{u}$ and therefore its $\delta_{u}$ and $\tau_{u}^{\max }$ has higher importance than the studies about $\tau_{c}$. For instance, during preliminary investigations of our algorithms, we understood that if $\tau_{u}$ is initially set to 1 and $\tau_{u}^{\max }>1$, the algorithm tends to grow a handful of huge models encompassing most of the strings in $\mathcal{D}$ and few others composed of one string only. That is why we decided, for this investigation, to begin with $\tau_{u}=-1, \delta_{u}=0.5-$ i.e. no update actions for the first three iterations but just creations. To some extent, the behaviour we obtained by GNG would somewhat correspond to first spawning far away centroids, and subsequently scatter new/update existing ones. Should this be the real key strength of GNG then this could mean that $\tau_{u}$ could be automatically toggled (i.e. set to values greater than zero) once a certain portion of $\mathcal{D}$ has been assigned to models. Moreover, as it was highlighted in Section $6, \tau_{c}$ appears to be related to the string length statistics of the dataset at hand. Should this be also observed in the presence of other datasets, then it could be possible to make GNG hyperparameter-free. Another way to investigate whether GNG can indeed become hyperparameter-free is the application of it in supervised learning scenarios. By adding constraints on the returned ensemble size, GNG could autonomously set its $\tau_{c}, \tau_{u}$ to perform optimal clustering and modelling. Finally, further investigations on the effect that the size of n-grams history has on the performance of 
GNG (and other algorithms) is due. Another strategy would even correspond into making GNG, or even each model independently, to become able to chose their own $n$ value.

As explained in Section 1, our ultimate goal is the creation of a computational framework which helps identifying "average" usage for system (reliability) verification. The idea is to leverage usage models to identify (i.e. classify), from a given dataset, logs depicting normal behaviour, and then to re-execute them. This requires a high level of self-adaptation by the algorithm in charge of maintaining the usage models. We claim that GNG implements a computational approach which is much more prone to selfadaptation than those relying on pairwise distance matrices of string vectorisation since, unlike the latter two, it does not require any form of data preprocessing. Nevertheless, in order to achieve full GNG's self-adaptation, future work could focus on: (1) understanding whether and how to re-initialise GNG's hyperparameters each time new strings are to be processed; (2) re-design the algorithm to accept data-streams rather than batches; (3) allowing the possibility to discard old models which have not been updated in long time; (4) allowing the possibility to merge models which perhaps started far away but after several update actions now manifest an excessively high degree of overlap; (5) allow the existence of unassigned strings (i.e. line 25 in the pseudocode) rather than coercively assign them to potentially wrong models. Ultimately, we envision GNG as an algorithm which, given a (stream) dataset, automatically sets its hyperparameters - even $n$, or different $n$ s for different models - performs its clustering/modelling computation repeatedly until its internal entropy converges, is capable of merging/splitting/discarding models, and finally is also capable of maintaining a genealogy of models, so that it can even backtrack its decisions and possibly highlight how system usage evolved through time.

The performance target of our investigation focussed on minimising average entropy scores, given a set of algorithms and hyperparameters. While this gives insights on the computational efficiency of our GNG algorithm, it does not provide insights on whether the retrieved models are semantically correct. In order to leverage the model for the identification of "average" system usage, we necessarily need to verify whether or not the models make sense from the viewpoint of domain experts. However, models such as the one shown in Figure 5, let alone bigger ones, are not easy to grasp by domain experts, as we witnessed during early stages of our research. An alternative approach, given the probabilistic nature of our mod-

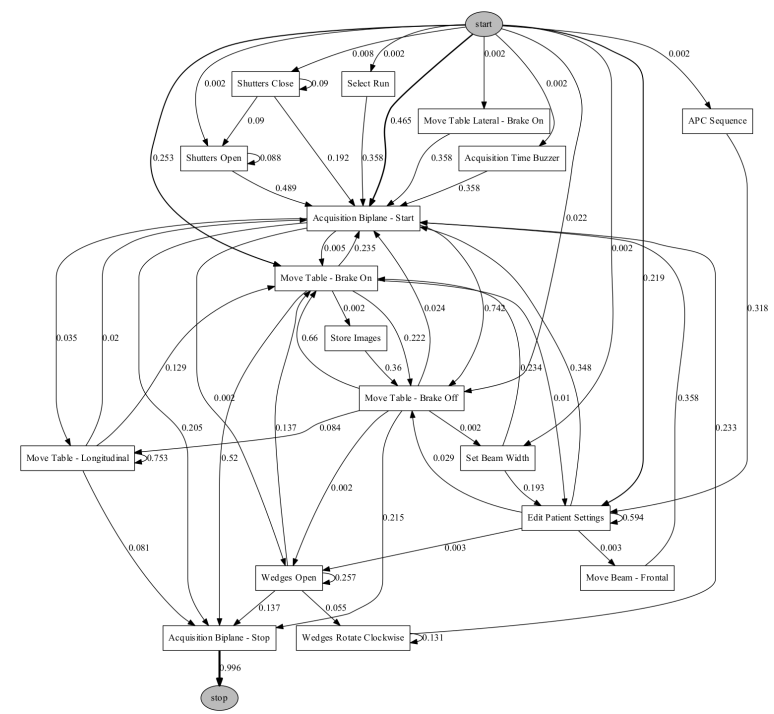

Figure 5: An example of a n-gram model obtained via GNG with $\tau_{c}=20$. It is composed of 20 nodes and 56 edges and represents a cluster of 160 strings, 55 of which unique. The values along the edges correspond to the conditional probabilities. The values do not sum up to one because of Kneser-Ney smoothing.

els, is to traverse them based on such probabilities and record traces of visited nodes. The traces, which we would call synthetic bolus chase procedures, could then be evaluated by domain experts. The trace evaluation would semantically validate the goodness of the usage models, albeit in an indirected and partial way. To pursue this investigation we launched a crowd-sourced experiment in which experts from different domains (e.g. engineering, clinical, research) are asked to observe traces - which might either be synthetic or real ones extracted directly from raw data - and to judge whether they would occur during clinical practice of Bolus Chase, much like a Turing test for artificial intelligence. The experiment is expected to run until Spring 2019.

\section{CONCLUSIONS}

Connected high-tech systems allow the gathering of operational data at unprecedented volumes. A direct benefit of this is the possibility to leverage data to draw usage models, in order to shorten the gap between how a system is designed to operate and how it is actually used. Usage models are beneficial for system reliability verification, part of system verification testing, in which knowledge of "normal" system behaviour is required. Our ultimate goal is the design of a computational framework which helps identifying "average" usage for system verification. We in- 
terpreted the task of modelling usage behaviours as string clustering/modelling task. We relied on n-gram modelling for the representation of usage models one n-gram model per retrieved cluster - and considered three algorithmic approaches to string clustering. The first two are based on the most commonly used $k$-means and complete-link and rely on either the transformation of the string data into vectorised forms, or the use of pairwise string-based similarity measures. We proposed a third approach and its related algorithm, called Growing N-Grams, which builds new or updates existing n-gram models based on string-model similarities and chain probabilities computed given the models, hence by avoiding any form of data pre-processing. We conducted an empirical evaluation of our approach based on a dataset representing sub-sequences of the Bolus chase procedure, a technique in peripheral arteriography, performed on Philips Healthcare's Image Guided Therapy interventional systems. The gathered results suggest that Growing N-Grams can return cluster compositions with lower entropy scores of classification, which indicate the algorithm's ability to retrieve different usage behaviours.

Growing N-Grams appears to be more prone to self-adaptation than other algorithms considered in our study, which also implies it could be better fit for our ultimate goal. We also highlighted a plethora of future work which would ultimately lead to the definition of a fully adaptive algorithm.

\section{ACKNOWLEDGEMENTS}

The research is carried out as part of the ITEA3 14035 REFLEXION project under the responsibility of the Embedded Systems Innovation by TNO (ESI) with Royal Philips as carrying industrial partner. The REFLEXION research is supported by the Netherlands Organisation for Applied Scientific Research TNO and Netherlands Ministry of Economic Affairs.

\section{REFERENCES}

Ackroyd, M. (1980). Isolated word recognition using the weighted levenshtein distance. IEEE Transactions on Acoustics, Speech, and Signal Processing, 28(2):243244.

Aggarwal, C. C. and Zhai, C. (2012). A survey of text clustering algorithms. In Mining text data, pages 77-128. Springer.

Anderberg, M. R. (1973). Cluster analysis for applications. Technical report, Office of the Assistant for Study Support Kirtland AFB N MEX.

Andrews, N. O. and Fox, E. A. (2007). Recent developments in document clustering.

Balabantaray, R. C., Sarma, C., and Jha, M. (2015). Document clustering using k-means and k-medoids. arXiv preprint arXiv:1502.07938.

Bengio, Y., Ducharme, R., Vincent, P., and Jauvin, C. (2003). A neural probabilistic language model. Journal of machine learning research, 3(Feb):1137-1155.

Bishop, C., Bishop, C. M., et al. (1995). Neural networks for pattern recognition. Oxford university press.

Brown, P. F., Desouza, P. V., Mercer, R. L., Pietra, V. J. D., and Lai, J. C. (1992). Class-based n-gram models of natural language. Computational linguistics, 18(4):467-479.

Buchta, C., Kober, M., Feinerer, I., and Hornik, K. (2012). Spherical k-means clustering. Journal of Statistical Software, 50(10):1-22.

Bungum, L. and Gambäck, B. (2012). Efficient ngram language modeling for billion word webcorpora. In Proceedings of the 8th International Conference on Language Resources and Evaluation, pages 6-12.

Chen, S. F. and Goodman, J. (1999). An empirical study of smoothing techniques for language modeling. Computer Speech \& Language, 13(4):359-394.

Cutting, D. R., Karger, D. R., Pedersen, J. O., and Tukey, J. W. (2017). Scatter/gather: A cluster-based approach to browsing large document collections. In ACM SIGIR Forum, volume 51, pages 148-159. ACM.

da Cruz Nassif, L. F. and Hruschka, E. R. (2013). Document clustering for forensic analysis: an approach for improving computer inspection. IEEE transactions on information forensics and security, 8(1):46-54.

Dai, A. M., Olah, C., and Le, Q. V. (2015). Document embedding with paragraph vectors. arXiv preprint arXiv:1507.07998.

Dhillon, I. S. and Modha, D. S. (2001). Concept decompositions for large sparse text data using clustering. Machine learning, 42(1-2):143-175.

Duda, R. O. and Hart, P. E. (1973). Pattern classification and scene analysis. A Wiley-Interscience Publication, New York: Wiley, 1973.

Ismail, S. and Rahman, M. S. (2014). Bangla word clustering based on n-gram language model. In Electrical Engineering and Information \& Communication Technology (ICEEICT), 2014 International Conference on, pages 1-5. IEEE. 
Jain, A. K. and Dubes, R. C. (1988). Algorithms for Clustering Data. Prentice-Hall, Inc., Upper Saddle River, NJ, USA.

Jaro, M. A. (1989). Advances in record-linkage methodology as applied to matching the 1985 census of tampa, florida. Journal of the American Statistical Association, 84(406):414-420.

Jurafsky, D. and Martin, J. H. (2014). Speech and language processing, volume 3. Pearson London.

Kešelj, V., Peng, F., Cercone, N., and Thomas, C. (2003). $\mathrm{N}$-gram-based author profiles for authorship attribution. In Proceedings of the conference pacific association for computational linguistics, PACLING, volume 3, pages 255-264.

Kim, Y. (2014). Convolutional neural networks for sentence classification. arXiv preprint arXiv:1408.5882.

Kiros, R., Zhu, Y., Salakhutdinov, R. R., Zemel, R., Urtasun, R., Torralba, A., and Fidler, S. (2015). Skipthought vectors. In Advances in neural information processing systems, pages 3294-3302.

Kneser, R. and Ney, H. (1995). Improved backing-off for $\mathrm{m}$-gram language modeling. In icassp, volume 1, page $181 \mathrm{e} 4$.

Lau, J. H. and Baldwin, T. (2016). An empirical evaluation of doc2vec with practical insights into document embedding generation. CoRR, abs/1607.05368.

Le, Q. and Mikolov, T. (2014). Distributed representations of sentences and documents. In International Conference on Machine Learning, pages 1188-1196.

Lesher, G. and Rinkus, G. (2002). Leveraging word prediction to improve character prediction in a scanning configuration. In Proceedings of the RESNA 2002 Annual Conference, pages 90-92.

Lesher, G. W., Moulton, B. J., and Higginbotham, D. J. (1998). Optimal character arrangements for ambiguous keyboards. IEEE Transactions on Rehabilitation Engineering, 6(4):415-423.

Levenshtein, V. I. (1966). Binary codes capable of correcting deletions, insertions, and reversals. In Soviet physics doklady, volume 10, pages 707-710.

Lu, S.-Y. and Fu, K. S. (1978). A sentence-to-sentence clustering procedure for pattern analysis. IEEE Transactions on Systems, Man, and Cybernetics, 8(5):381389.

Mahdavi, M. and Abolhassani, H. (2009). Harmony kmeans algorithm for document clustering. Data Mining and Knowledge Discovery, 18(3):370-391.

Markov, A. (1971). Extension of the limit theorems of probability theory to a sum of variables connected in a chain.

Marzal, A. and Vidal, E. (1993). Computation of normalized edit distance and applications. IEEE transactions on pattern analysis and machine intelligence, 15(9):926-932.

Miao, Y., Kešelj, V., and Milios, E. (2005). Document clustering using character n-grams: a comparative evaluation with term-based and word-based clustering. In Proceedings of the 14th ACM international conference on Information and knowledge management, pages 357-358. ACM.
Mikolov, T., Chen, K., Corrado, G., and Dean, J. (2013a). Efficient estimation of word representations in vector space. arXiv preprint arXiv:1301.3781.

Mikolov, T., Sutskever, I., Chen, K., Corrado, G. S., and Dean, J. (2013b). Distributed representations of words and phrases and their compositionality. In Advances in neural information processing systems, pages 31113119.

Pettersson, E., Megyesi, B., and Nivre, J. (2013). Normalisation of historical text using context-sensitive weighted levenshtein distance and compound splitting. In Proceedings of the 19th Nordic Conference of Computational Linguistics (NODALIDA 2013); May 22-24; 2013; Oslo University; Norway. NEALT Proceedings Series 16, number 085, pages 163-179. Linköping University Electronic Press.

Raghavan, V. V. and Birchard, K. (1979). A clustering strategy based on a formalism of the reproductive process in natural systems. In ACM SIGIR Forum, volume 14, pages 10-22. ACM.

Rajalingam, N. and Ranjini, K. (2011). Hierarchical clustering algorithm-a comparative study. International Journal of Computer Applications, 19(3):42-46.

Rehurek, R. and Sojka, P. (2010). Software framework for topic modelling with large corpora. In In Proceedings of the LREC 2010 Workshop on New Challenges for NLP Frameworks. Citeseer.

Salton, G. (1989). Automatic text processing: The transformation, analysis, and retrieval of. Reading: AddisonWesley.

Siivola, V. and Pellom, B. L. (2005). Growing an n-gram language model. In Ninth European Conference on Speech Communication and Technology.

Steinbach, M., Karypis, G., Kumar, V., et al. (2000). A comparison of document clustering techniques. In $K D D$ workshop on text mining, volume 400, pages 525-526. Boston.

Tai, K. S., Socher, R., and Manning, C. D. (2015). Improved semantic representations from tree-structured long short-term memory networks. arXiv preprint arXiv:1503.00075.

Wurzenberger, M., Skopik, F., Landauer, M., Greitbauer, P., Fiedler, R., and Kastner, W. (2017). Incremental clustering for semi-supervised anomaly detection applied on log data. In Proceedings of the 12th International Conference on Availability, Reliability and Security, Article, number 31.

Xu, J., Wang, P., Tian, G., Xu, B., Zhao, J., Wang, F., and Hao, H. (2015). Short text clustering via convolutional neural networks. In VS@HLT-NAACL, pages 62-69.

Yujian, L. and Bo, L. (2007). A normalized levenshtein distance metric. IEEE transactions on pattern analysis and machine intelligence, 29(6):1091-1095.

Zhang, X., Zhao, J., and LeCun, Y. (2015). Characterlevel convolutional networks for text classification. In Advances in neural information processing systems, pages 649-657.

Zhao, Y., Karypis, G., and Fayyad, U. (2005). Hierarchical clustering algorithms for document datasets. Data mining and knowledge discovery, 10(2):141-168. 